\title{
Comparison of uveitis in the course of juvenile idiopathic arthritis with isolated uveitis in children - own experiences
}

\author{
Agnieszka Zygmunt ${ }^{1}$, Joanna Lipińska ${ }^{1}$, Małgorzata Biernacka-Zielińska², Ewa Lipiec ${ }^{3}$, \\ Anna Niwald ${ }^{3}$, Elżbieta Smolewska ${ }^{1}$
}

${ }^{1}$ Department of Pediatric Rheumatology, Medical University of Łodz, Maria Konopnicka Memorial Teaching Hospital No. 4 in Lodz, Poland

${ }^{2}$ Clinic of Pediatric Cardiology and Rheumatology, Maria Konopnicka Memorial Teaching Hospital No. 4 in Lodz, Poland

${ }^{3}$ Department of Pediatric Ophthalmology, Maria Konopnicka Memorial Teaching Hospital No. 4 in Lodz, Poland

\begin{abstract}
Objectives: Uveitis and juvenile idiopathic arthritis (JIA) relatively often coexist. Inflammatory changes in the anterior segment of the eye are the most common extra-articular symptom in children with JIA, and JIA is, in turn, the main systemic cause of anterior uveitis in children. The aim of our study was to compare the course of anterior uveitis accompanying JIA and isolated uveitis.

Material and methods: We analyzed 25 children with JIA and uveitis (group I) and 28 children with isolated uveitis (group II). The study population was retrospectively selected from the patients treated in our center in the years 1998-2016 through a search of the hospital database. All data were presented as descriptive statistics.

Results: In group I there was a higher percentage of girls than in group II (64\% vs. 50\%) and uveitis occurred at a significantly younger age (8.7 years vs. 11.6). Patients from group I more often presented with immunological abnormalities (positive antinuclear antibodies or HLA-B27 antigen). The majority of children from group I developed uveitis prior to (44\%) or simultaneously with (20\%) arthritis. In patients who first presented with uveitis, arthritis appeared on average after 28 months (median 12 months). In children in whom arthritis developed first, uveitis appeared on average after 51 months (median 36 months). In some patients the time interval between the involvement of these two organs was as long as 9-10 years. Four children from group I and three from group II were qualified for biological treatment.

Conclusions: The results of our analysis indicate the need for constant cooperation between the pediatric rheumatologist and the ophthalmologist. Although the risk of uveitis in JIA decreases with the disease duration, in some cases this complication can develop after many years. Children with present antinuclear antibodies, at younger age and of female gender should be subject to particularly close observation.
\end{abstract}

Key words: juvenile idiopathic arthritis, uveitis, isolated uveitis.

\section{Introduction}

Chronic inflammatory joint disease can begin at any age, including during childhood. Juvenile idiopathic arthritis (JIA) is the most common of these disorders. This term comprises a heterogeneous group of chronic arthritis of unknown etiology that begin before 16 years of age. If untreated, the disease most often leads to disability of the musculoskeletal system. However, this is not the only real threat to the young patients, as in the course of inflammatory joint diseases in children, the

Address for correspondence:

Agnieszka Zygmunt, Department of Pediatric Rheumatology, Medical University of Lodz, Maria Konopnicka Memorial Teaching Hospital No. 4, 36/50 Sporna St., 91-738 Lodz, Poland, e-mail: agnieszka2zygmunt@gmail.com

Submitted: 29.03.2018; Accepted: 7.05.2018 

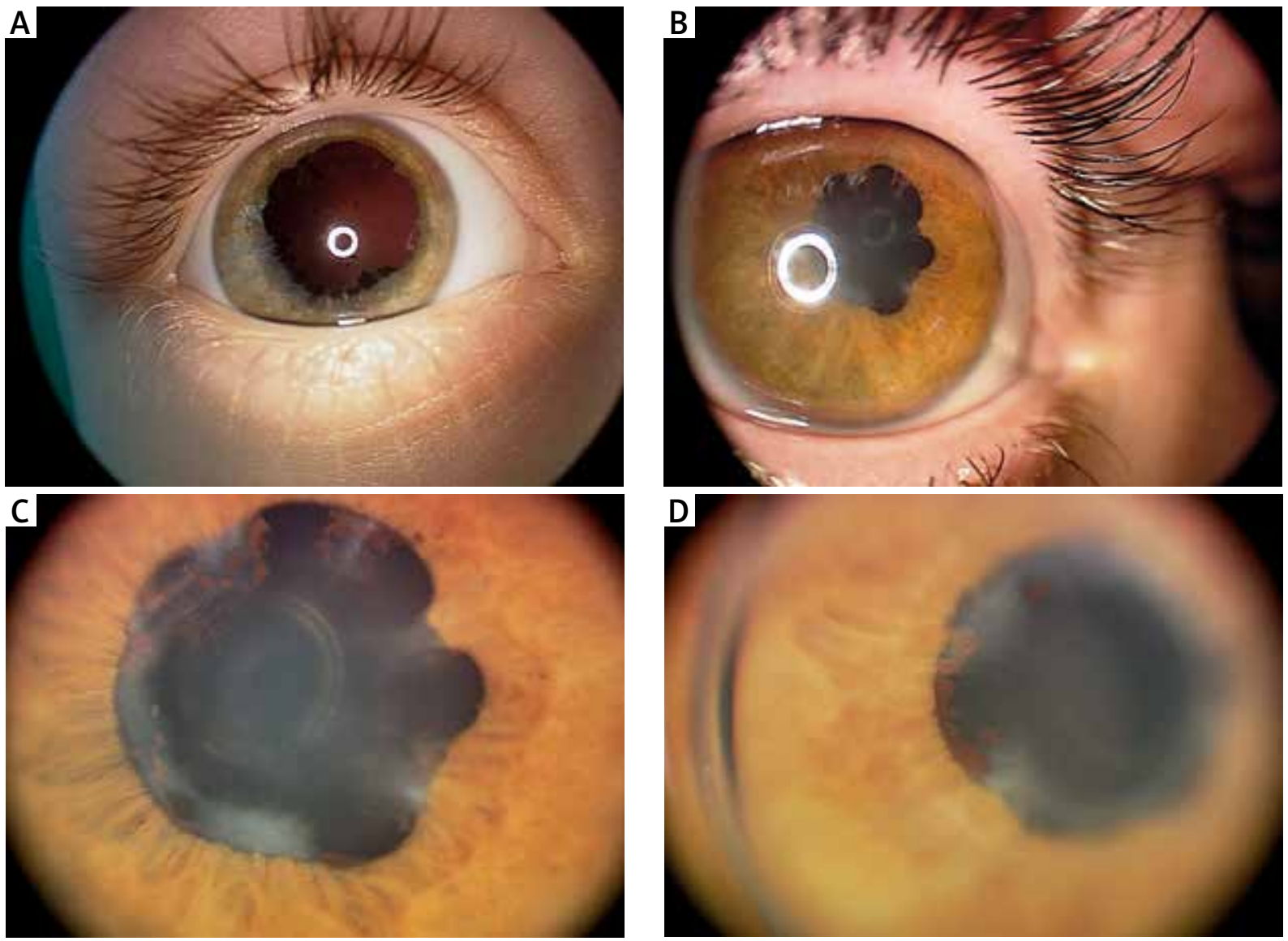

Fig. 1. Changes in the anterior segment of the eye in the children with uveitis (authors' own material). (A) Pupil de-rounding, few lenticular-iris adhesions, traces of old adhesions on the frontal lobe of the lens in the course of the anterior uveitis. (B) Numerous lenticular-iris adhesions and dye grains on the frontal lobe of the lens in the course of the anterior uveitis. (C, D) Pupil de-rounding, plane lenticular-iris adhesions, dye grains on the front lens bag, opacity of the lens in the course of anterior uveitis.

involvement of the anterior segment of the eye in the form of uveitis may also occur [1-4] (Fig. 1).

There are several subtypes of JIA - the oligoarticular form of JIA and enthesitis-related arthritis are characterized by the highest risk of eye involvement. The first subtype is especially dangerous, since the uveitis accompanying it has an insidious, chronic nature. In the second subtype acute uveitis predominates. Inflammatory changes in the anterior segment of the eye are the most common extra-articular symptom in children with $J A$, and $J \mid A$ is, in turn, the main systemic cause of anterior uveitis in children.

The aim of our study was to analyze the disease course in children with JIA and anterior uveitis, who are or were under the care of our center. We also wanted to compare this population with a group of children with isolated uveitis.

\section{Material and methods}

We analyzed 25 children with JIA and uveitis (group I). The reference group consisted of 28 children with isolated uveitis (group II). The study population was retrospectively selected from the patients treated in our University Hospital in the years 1998-2016 through a search of the database of the Department of Pediatric Rheumatology and the Department of Pediatric Ophthalmology. Their charts were then screened for demographic data, medical history, diagnostic testing and medications taken. The 1997 International League of Associations of Rheumatology (ILAR) criteria for JIA subgroups were used to define the type of juvenile arthritis. The diagnosis of isolated uveitis was made when no extraocular symptoms could be found and after exclusion of other causes of uveitis, especially infections. 
All data were presented as descriptive statistics (mean \pm standard deviation and percentages). The $\chi^{2}$ test was used to compare the variables. A $p$-value $<0.05$ was considered statistically significant.

\section{Results}

\section{Characteristics of examined groups}

Girls prevailed in the group of children with JIA and uveitis ( $n=16$, which accounted for $64 \%$ ). In the second group we did not observe predominance of either gender (14 girls and 14 boys). The most common form of JA was the oligoarticular beginning ( $n=19$, which accounted for $76 \%$ ), 4 patients (16\%) were diagnosed with an enthesitis-related arthritis and 2 with seronegative polyarticular disease.

Chronic arthritis in our patients appeared between 2 and 16 years of age (mean $8.18 \pm 5.06$ years), with two distinct peaks of the disease onset: between 2 and 5 years of age $(n=11)$ and between 13 and 16 years $(n=8)$. In slightly less than half of the children from group I eye symptoms preceded joint involvement ( $n=11$, which accounted for 44\%), in 5 children (20\%) articular and ocular symptoms occurred simultaneously, and in the remaining 9 children (36\%) uveitis joined arthritis at a certain time point.

In the group of children who first presented with uveitis, joint symptoms appeared after an average of 28 months ( \pm 35 months), with a median of 12 months. In the group of children in whom arthritis took place first, ocular symptoms appeared after an average of 51 months ( \pm 37 months), with a median of 36 months. In both groups, the spread of this period was similar and ranged from a few months to 9-10 years. The longest intervals between the onset of ocular and articular symptoms concerned 1 patient who first presented with uveitis and secondly with arthritis (4 years of age vs. 14 years of age) and 3 children in whom arthritis ap- peared first, and later uveitis developed in the acute form (age at JIA onset -2 years, 2 years, 4 years and the age of uveitis onset - 9 years, 10 years, 14 years, respectively).

In patients with isolated uveitis the disease was diagnosed between 3 and 17 years of age (mean 11.57 \pm 4.14 years).

All patients were treated with corticosteroids and/or disease-modifying antirheumatic drugs (DMARDs). The most frequently used DMARDs in the group of children with JIA were methotrexate (66\%), sulfasalazine (46\%) and cyclosporine (38\%); half of these patients received combined therapy - with 2 (42\%) or 3 (8\%) DMARDs. Patients with isolated uveitis, apart from corticosteroids, were treated only with either cyclosporine or methotrexate. Following the inadequate response to conventional treatment, 4 children from group I and 3 from group II were qualified for biological treatment.

\section{Comparison of children with uveitis in the course of JIA (group I) and isolated uveitis (group II)}

The main differences between group I and group II are shown in Table I. In the group of children with joint disease and uveitis there was a higher percentage of girls than in the population of patients with isolated uveitis (64\% vs. $50 \%$ ). Inflammatory changes within the eye occurred at a significantly younger age in group I than in group II (8.72 years of age \pm 4.64 vs. $11.57 \pm 4.14$ ). Patients with JIA much more often presented with immunological abnormalities. In $56 \%$ of the patients positive antinuclear antibodies were observed, often at a very high titer, whereas in the group with isolated uveitis antinuclear antibodies were present only in 2 cases (7\%). Moreover, a positive HLA-B27 antigen was also found far more often in group I than in group II (36\% vs. 10\%). In none of the groups were antibodies to citrullinated peptides (ACPA) detected, while in 1 patient with JIA and uveitis

Table I. Comparison of children with co-existing juvenile idiopathic arthritis and uveitis (group I) and children with isolated uveitis (group II)

\begin{tabular}{|lccc|}
\hline Parameters & Group I $(n=25)$ & Group II $(n=28)$ & $p$-value \\
\hline Age of uveitis onset (in years) & $8.72 \pm 4.64$ & $11.57 \pm 4.14$ & 0.027 \\
\hline Female gender & $16(64 \%)$ & $14(50 \%)$ & NS \\
\hline Presence of antinuclear antibodies & $14(56 \%)$ & $2(7 \%)$ & $p<0.001$ \\
\hline Positive rheumatoid factor & 1 & 0 & NS \\
\hline Positive HLA-B27 antigen & $9(36 \%)$ & $3(11 \%)$ & NS $(p=0.06)$ \\
\hline Bilateral uveitis & $11(44 \%)$ & $11(39 \%)$ & NS \\
\hline Recurrent uveitis & $15(60 \%)$ & $19(68 \%)$ & NS \\
\hline
\end{tabular}

NS - not significant 
a positive rheumatoid factor (RF) was found, but only in a single assay, not confirmed in other, repeated assessments, which ultimately did not allow the diagnosis of RF-positive JIA to be established.

In both groups inflammatory changes in the eyes were slightly more frequently unilateral ( $56 \%$ of children in group I and $61 \%$ of children in group II). In most cases, the uveitis was recurrent ( $60 \%$ of patients in group I and $68 \%$ of patients in group II).

\section{Discussion}

According to data from the literature the incidence of uveitis in children with $\mathrm{IA}$ is about $10-25 \%[5,6]$. The majority of these patients develop inflammatory changes in the eye within the first 4 years of the disease and this period is generally considered as requiring increased vigilance [5, 6]. Our observations suggest, however, that there is a need for scrupulous ophthalmological observation of patients even with longer lasting inflammatory processes of the joints. The mean time to the onset of ocular symptoms in the group of our patients with JIA falls at the end of a 4-year period of increased risk for developing uveitis, and in some children such complications appear even after 7-9 years. Blindness or significant impairment in visual acuity, which is the most unfavorable outcome of uveitis, can impair the quality of life of these patients much more than disability of the musculoskeletal system. Because of this, in terms of the results of our study, one should maintain full alert even after those four years. However, it is worth noting that the observations of some authors indicate that in cases of uveitis appearing within a short period after the onset of arthritis, its course is more severe and eye complications are more likely to develop [7-9]. This explains why close monitoring of the patient within the first year after the diagnosis of JIA is so crucial.

Hoeve et al. [10] pointed out the biphasic character of occurrence of inflammatory lesions in the eyes in children with JIA, with the first bout in early childhood and the second one during adolescence. Based on our observations it cannot be excluded that such a biphasic course may have a mixed character - one of those bouts may affect the eyes, while another may affect the joints, in any sequence. Both of these presentations of the dis ease may have similar underlying mechanisms, and puberty may be a factor provoking autoimmune disorders in the organism.

Surprisingly, a large group of children presented with inflammatory changes within the anterior segment of the eye prior to arthritis - it concerned almost half of our patients with JIA. In some patients, it was the first routine ophthalmologic examination after the diagno- sis of arthritis that showed old changes within the eye resulting from the prior, silent uveitis, which is also described in the literature [11]. However, most of the children remained under the care of the ophthalmologist earlier and, according to our observations, this is the group of increased risk of inflammatory disease of the joints. This points to the need for close rheumatological observation of those patients, as well as performing basic laboratory screening for autoimmune abnormalities in children with isolated uveitis. On its basis, one can distinguish patients with an increased risk of arthritis in the future, which include the presence of antinuclear antibodies or positive antigen HLA-B27. Also in this group, we observed a long possible interval between the onset of ocular and articular symptoms, as it was on average approximately 2.5 years and it reached a maximum of years.

In the literature, it is emphasized that patients with IIA accompanied by uveitis are significantly younger at the time of initial diagnosis of the joint disease (e.g. 2.8 years vs. 7.7 years in the study by Angeles-Han et al.) [6]. In our study, we observed that a similar relationship can be found when comparing the age at diagnosis of children with uveitis and concomitant JIA and children with isolated uveitis. In the first subgroup there was a high percentage (32\%) of children in the youngest age group (2-5 years), while there was only 1 patient with isolated uveitis in this age group. Not all children who were diagnosed with JIA at the youngest age also developed uveitis in this age range, however; it concerned only 2 patients, and in the rest of them ( $24 \%$ of the group) diagnosis of uveitis was also established before the age of 5 . It is also noticeable that in the youngest age group the interval between uveitis and arthritis was generally shorter than in older children, but, on the other hand, the 3 children with the longest interval were diagnosed with JIA before the age of 5 . On the basis of the very rare occurrence of isolated uveitis in the youngest age group observed in our study, it may be assumed that children who developed uveitis at this age without prior inflammation of the joints are at high risk for developing such changes within the next few years, particularly if positive antinuclear antibodies are present in such a patient. Similar observations were made by Manzotti et al. [12].

Like in studies by other authors [5, 13, 14], the oligoarticular form of the disease also prevailed in our group of children with JIA and uveitis. This predominance was most marked in the youngest age group. During adolescence, apart from oligoarthritis, we often observed enthesitis-related arthritis - it was a subgroup in which quite a large percentage of patients presented with positive antigen HLA-B27 and uveitis was acute in character. 
A similar distribution of subtypes of JIA and uveitis is described in the available literature [15]. In more than half of children from group I positive antinuclear antibodies were detected. It is smaller percentage than in some other reports (70-90\%) [13], but after including in the analysis only children in the first decade of life, this number rises to almost $80 \%$. Many patients had very high titers of antinuclear antibodies, although sometimes in the literature it is emphasized that the height of the titer does not correlate with the risk of developing an inflammatory process in the eye either with the severity of its course [13]. Similarly as in observations of other authors, children with isolated uveitis rarely presented with positive antinuclear antibodies [12].

In the whole analyzed group of children with JIA and uveitis there were only 2 patients who during our observation developed significant impairment of visual acuity, while no child developed blindness. In some reports, especially before the year 1990, when more effective pharmacotherapy of JIA was introduced, such complications were described more frequently [16]. In a study conducted in the USA by Angeles-Han et al., such a dramatic course of the disease concerned mainly the Afro-American population, which was associated by the authors not only with a potentially worse course of the disease itself, but also with possible worse access to health care [6]. Based on our research we can conclude that the ophthalmic care of children with JIA in our region is conducted effectively, and despite the occurrence of this extra-articular manifestation of the disease, it rarely leads to permanent damage of the structures within the eye.

Treatment of uveitis is based on the use of local and general glucocorticoids, mydriatics or, in severe cases, immunosuppression (methotrexate, azathioprine, cyclosporine A). Both among children with uveitis accompanying IA and isolated uveitis there were patients who did not respond to conventional treatment and were qualified for biological therapy. Such treatment resulted in the relief of symptoms from the joints and/or the eye. Similar experiences are described by other authors $[17,18]$.

In the literature it is suggested that such treatment of uveitis should be conducted for 12-24 months. However, during monitoring the disease course after the end of biological treatment we observed that children with isolated uveitis maintained long remissions, lasting even several years, while patients with uveitis accompanying JIA in most cases developed relapses of the disease several weeks after stopping the biological treatment. They concerned mostly articular symptoms, although in 1 patient both the articular and the ocular changes reappeared after discontinuation of biological treatment. Many authors have also pointed out that the clinical course of uveitis with JIA is more severe than that of idiopathic uveitis, with more relapses and complications $[2,12,19]$. A high percentage of early relapses following anti-TNF therapy discontinuation is also reported in the population of patients with JIA without eye involvement [20, 21].

In the literature it is emphasized that in the future, advances in the management of uveitis, apart from biological therapy, should be based on prospective population registries analysis and biomarker studies to predict the type of onset of JIA, prognosis, organ damage and therapeutic response.

\section{Conclusions}

The results of our analysis indicate the need for constant cooperation between the pediatric rheumatologist and ophthalmologist. Patients with JIA require periodic ophthalmologic check-up and those with inflammation of the anterior part of the vascular membrane of the eye require rheumatologic observation. Although the risk of uveitis in JIA decreases with the disease duration, in some cases, such a complication can develop after many years. Children with positive antinuclear antibodies, at a younger age and of female gender should be subject to particularly close observation.

The authors declare no conflict of interest.

\section{References}

1. Heiligenhaus A, Michels H, Schumacher C, et al. German Ophthalmological Society; Society for Childhood and Adolescent Rheumatology; German Society for Rheumatology. Evidence-based, interdisciplinary guidelines for anti-inflammatory treatment of uveitis associated with juvenile idiopathic arthritis. Rheumatol Int 2012; 32: 1121-1133.

2. Bou R, Adán A, Borrás F, et al. Clinical management algorithm of uveitis associated with juvenile idiopathic arthritis: interdisciplinary panel consensus. Rheumatol Int 2015; 35: 777-785.

3. Yu HH, Chen PC, Wang LC, et al. Juvenile idiopathic arthritis-associated uveitis: a nationwide population-based study in Taiwan. PLoS One 2013; 8: 1-7.

4. Angeles-Han ST, Pelajo CF, Vogler LB, et al. CARRA Registry Investigators. Risk markers of juvenile idiopathic arthritis-associated uveitis in the Childhood Arthritis and Rheumatology Research Alliance (CARRA) Registry. J Rheumatol 2013; 40: 2088-2096.

5. Vitale AT, Graham E, de Boer JH. Juvenile Idiopathic Arthritis-Associated Uveitis: Clinical Features and Complications, Risk Factors for Severe Course, and Visual Outcome. Ocul Immunol Inflamm 2013; 21: 478-485.

6. Angeles-Han ST, McCracken C, Yeh S, et al. Characteristics of a cohort of children with Juvenile Idiopathic Arthritis and JIA-associated Uveitis. Pediatr Rheumatol Online J 2015; 13: 19. 
7. Clarke SL, Sen ES, Ramanan AV. Juvenile idiopathic arthritis-associated uveitis. Pediatr Rheumatol Online J 2016; 14: 27.

8. Woreta F, Thorne JE, Jabs DA, et al. Risk factors for ocular complications and poor visual acuity at presentation among patients with uveitis associated with juvenile idiopathic arthritis. Am J Ophthalmol 2007; 143: 647-655.

9. Campanilho-Marques R, Bogas M, Ramos F, et al. Prognostic value of antinuclear antibodies in juvenile idiopathic arthritis and anterior uveitis. Results from a systematic literature review. Acta Reumatol Port 2014; 39: 116-122.

10. Hoeve M, Kalinina AV, Schalij-Delfos NE, et al. The clinical course of juvenile idiopathic arthritis-associated uveitis in childhood and puberty. Br J Ophthalmol 2012; 96: 852-856.

11. Rodriguez-Garcia A. The Importance of an Ophthalmologic Examination in Patients with Juvenile Idiopathic Arthritis. Reumatol Clin 2015; 11: 133-138.

12. Manzotti F, Orsoni JG, Zavota L, et al. Autoimmune uveitis in children: clinical correlation between antinuclear antibody positivity and ocular recurrences. Rheumatol Int 2002; 21 : 127-132.

13. Heiligenhaus A, Minden K, Föll D, et al. Uveitis in juvenile idiopathic arthritis. Dtsch Arztebl Int 2015; 112: 92-100.

14. Angeles-Han ST, Yeh S, Vogler LB. Updates on the risk markers and outcomes of severe juvenile idiopathic arthritis-associated uveitis. Int J Clin Rheumtol 2013; 8: 1-20.
15. Moradi A, Amin RM, Thorne JE. The role of gender in juvenile idiopathic arthritis - associated uveitis. J Ophthalmol 2014; 2014: 461078.

16. Saurenmann RK, Levin AV, Feldman BM, et al. Prevalance, Risk Factors, and Outcome of Uveitis in Juvenile Idiopathic Arthritis. Arthritis Rheum 2007; 56: 647-657.

17. Semeraro F, Arcidiacono B, Nascimbeni G, et al. Anti-TNF therapy for juvenile idiopathic arthritis-related uveitis. Drug Des Devel Ther 2014; 8: 341-348.

18. Wells JM, Smith JR. Uveitis in juvenile idiopathic arthritis: recent therapeutic advances. Ophthalmic Res 2015; 54: 124127.

19. Heinz C, Mingels A, Goebel C, et al. Chronic Uveitis in Children with and without Juvenile Idiopathic Arthritis: Differences in Patient Characteristics and Clinical Course. J Rheumatol 2008; 35: 1403-1407.

20. Iglesias E, Torrente-Segarra V, Bou R, et al. Non-systemic juvenile idiopathic arthritis outcome after reaching clinical remission with anti-TNF- therapy: a clinical practice observational study of patients who discontinued treatment. Rheumatol Int 2014; 34: 1053-1057.

21. Postępski J, Kobusińska K, Olesińska E, et al. Clinical remission in juvenile idiopathic arthritis after termination of etanercept. Rheumatol Int 2013; 33: 2657-2660. 